\title{
Rare malformation of the left atrial appendage: report of a case
}

\begin{abstract}
In this report, we present a case of a rare malformation of the left atrial appendage. This finding was observed during the transesophageal echocardiogram performed on a patient diagnosed with supraventricular arrhythmia who was undergoing electrical cardioversion. This observed malformation of the left atrial appendage has not been previously described.
\end{abstract}

Keywords: left atrial appendage malformation, anatomy of the left atrial appendage
Volume 2 Issue 3 - 2017

\begin{abstract}
Jorge Tazar, Gerardo Marcos Palacio, Natali Arce Rojas, Pilar Haurigot, Myriam Espinosa Department of echocardiography, Instituto de Cardiología, Tucumán, Argentina
\end{abstract}

Correspondence: Jorge Tazar, Department of echocardiography, Instituto de Cardiología, Tucumán, San Miguel de Tucumán, Tucumán, Argentina, Tel +54038|4330389, Email jtazar@gmail.com

Received: November 14, 2017| Published: December 01, 2017
Abbreviations: AF, atrial fibrillation; AVB, atrioventricular block; ECV, electric cardio version; L, left atrium; LAA, left atrial appendage; TEE, transesophageal echocardiography

\section{Introduction}

In recent years, the anatomical study of the Left Atrial Appendage (LAA) has regained interest. Studies using cardiac nuclear magnetic resonance and multi slice computed tomography have described various morphologies of the LAA, namely: Cactus, chicken wing, windsock, and cauliflower. ${ }^{1}$ The hole that connects the LAA with the atrium has also been described. This route of entry is closely related to the arrival of the left superior pulmonary vein, the conduction system and the circumflex artery and can present different forms: Oval, circular, triangular or in drop. ${ }^{2}$ This report describes an LAA that manifests anatomical characteristics different from what is known.

\section{Case presentation}

A male patient, 66 years old, presents as risk factors a smoking habit that had ceased in the last 5 months. As a cardiovascular history, he had two previous admissions for supra ventricular arrhythmias, which were reversed in a timely manner with Electrical Cardioversion (ECV). The patient is admitted to the emergency room with symptoms of asthenia and adynamia of 72 hours of evolution. The 12 lead ECG showed an atrial flutter with a high degree of Atrioventricular Block (AVB), with a 5:1 conduction. It had a heart rate of 50 beats per minute (Figure 1). The patient received amiodarone 400mg./ day. He was hospitalized in a coronary unit to try a new ECV. Blood pressure: $150 / 80 \mathrm{mmHg}$. The exams of the other systems (respiratory, neurological, etc.) had no positive data. It is indicated to start anticoagulation with low molecular weight heparin, and a Transesophageal Echocardiogram (TEE) is requested to rule out cardio embolic source.

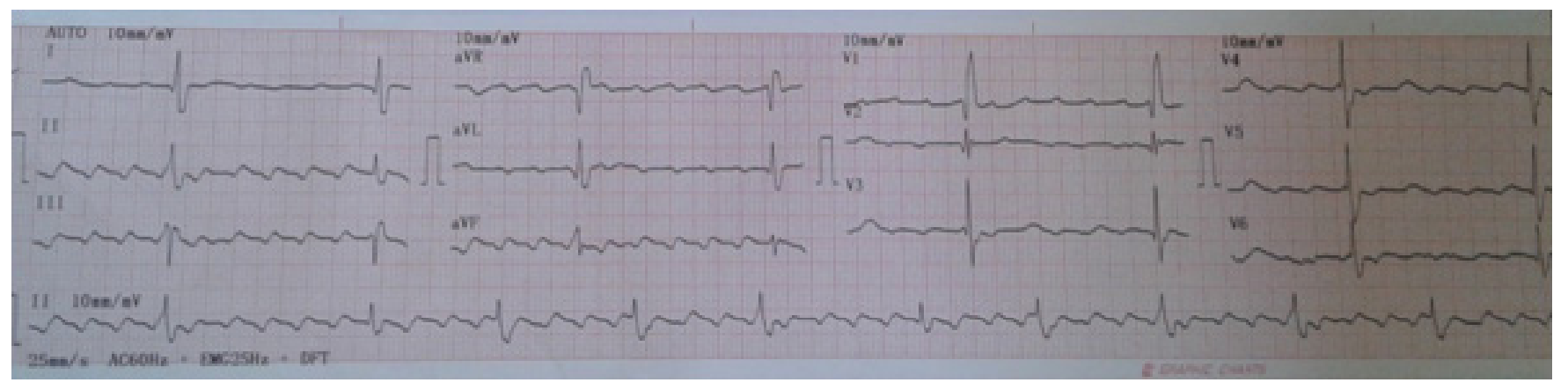

Figure I 12 lead ECG showing atrial flutter with a high degree of atrioventricular block (5:I conduction). it also shows complete right bundle-branch block and left anterior hemiblock.

\section{Echocardiographic findings}

The patient was in the left lateral decubitus under deep sedation and with oropharyngeal anesthesia with xylocaine, TEE was performed with a multi planar probe. At the middle esophageal level, with an angle of 87 degrees, it was possible to verify that the morphology of the LAA corresponded to the chicken wing type, with a size of 4.3 $\mathrm{cm} 2$ of area. It was noted that at the connection site between the LAA and the Left Atrium (LA) a "septum" that closure LAA. This structure showed no movement and when it was explored with color Doppler, it was found that it had a narrow perforation through which a highspeed bidirectional flow proceeded (Figure 2-5). In the interior of the appendage, there was no evidence of spontaneous contrast echo or thrombi. 


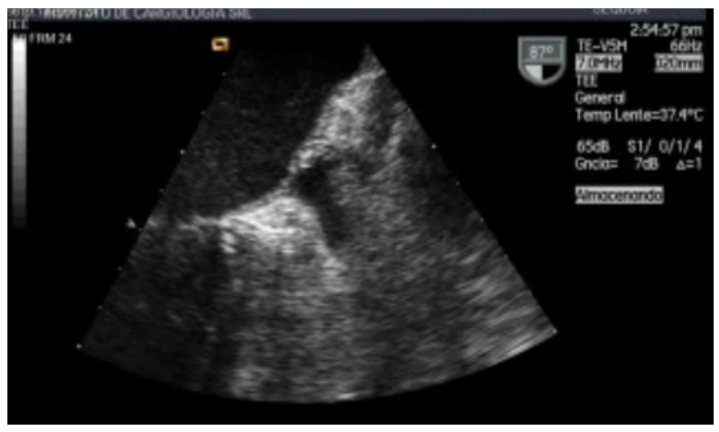

Figure 2 TEE at the mid-esophageal level, with an angle of 87 degrees. We can see the left atrial appendage (LAA) (thick arrow), and the septum that is in the connection site of the appendage with the left atrium (thin arrow).

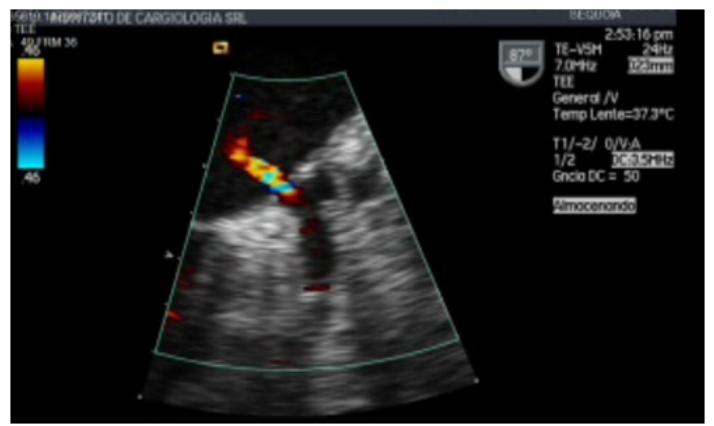

Figure 3 TEE in a projection located in the left atrial appendage using color doppler.The flow of emptying of the left atrial appendage can be observed.The color doppler shows a high speed flow.

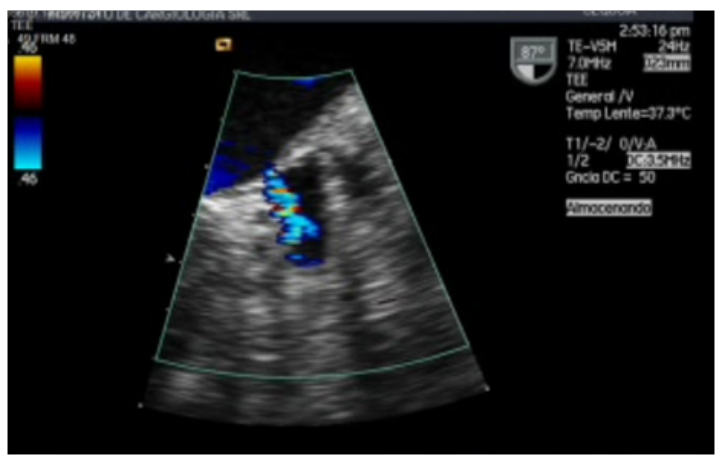

Figure 4 TEE in a projection located in the left atrial appendage using color doppler. The flow of filling of the left atrial appendage can be observed. The color doppler shows a high speed flow.

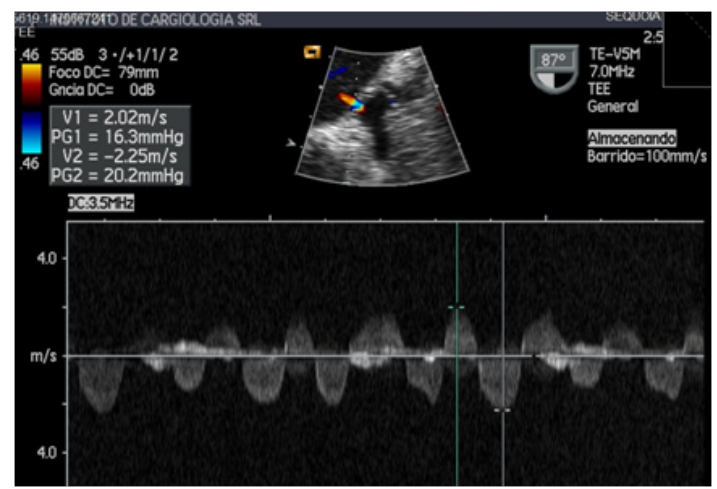

Figure 5 Spectral doppler showing high emptying and filling velocities of the left atrial appendage. You can see speeds above 2 meters per second.

\section{Discussion}

The LAA is an embryological remnant of the primordial atrium and is formed by the absorption of the primordial pulmonary veins and their branches. It is a glove-like structure, generally located between the anterior and lateral wall of the LA and whose tip is directed anteriorly and superiorly, overlapping the left edge of the right ventricle outflow tract or the pulmonary trunk and the birth of the left or circumflex coronary artery. It is not uncommon to find the tip of the LAA in the lateral and posterior direction. However, in a small percentage of hearts, the tip of the LAA passes behind the arterial pedicle to settle on the transverse sinus of the pericardium. ${ }^{2}$

The main importance of LAA is that it is the site where thrombi are most frequently formed in patients with Atrial Fibrillation (AF); these thrombi carry the risk of subsequent embolism. Necropsy studies have described that in $98 \%$ of the cases thrombi formed in the atrium of patients with AF are found in the LAA. ${ }^{3}$ Taking into account these precedents, for several decades, they have been considered the closure of the LAA as a treatment in the prevention of stroke. ${ }^{4}$ In the beginning, the closure was performed surgically, either through a split or an exclusion. However, in recent years, the exclusion of the LAA has been performed with percutaneous closure devices. Currently, there are several models and there are still others in research. ${ }^{5} \mathrm{~A}$ report about a malformation at the level of the left atrial appendage is presented. There is no previous evidence that refers to this type of anatomy of the LAA and for our consideration it would be a rare congenital anomaly of the same. Characterizing the real clinical impact of this finding is difficult in light of the current knowledge.

\section{Patient consent form}

The authors have the patient's permission for the presentation of the case report.

\section{Acknowledgements}

None.

\section{Conflict of interest}

The authors do not present any conflict of interest.

\section{References}

1. Di Biase L, Santangeli P, Anselmino M, et al. Does the left atrial appendage morphology correlate with the risk of stroke in patients with atrial fibrillation? Results from a multicenter study. J Am Coll Cardiol. 2012;60(6):531-538.

2. Beigel R, Wunderlich NC, Ho SY, et al. The left atrial appendage: Anatomy, function, and noninvasive evaluation. J Am Coll Cardiol Img. 2014;7(12):1251-1265.

3. Manning WJ, Weintraub RM, Waksmonski CA, et al. Accuracy of transesophageal echocardiography for identifying left atrial thrombi. A prospective, intraoperative study. Ann Intern Med. 1995;123(11):817822 .

4. Reddy V, Holmes D, Doshi S, et al. Safety of percutaneous left atrial appendage closure. Results from the watchman left atrial appendage system for embolic. Protection in patients with AF (PROTECT AF) clinical trial and the continued access registry. Circulation. 2011;123(4):417-424.

5. Kanderian A, Gillinov A, Petterson G, et al. Success of surgical left atrial appendage closure. Assessment by tranesophageal echocardiography. $J$ Am Coll Cardiol. 2008;52(11):924-929. 